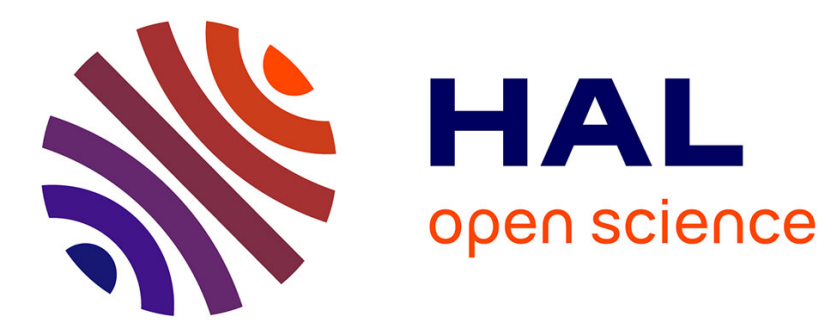

\title{
An unified view of multitude behavior, flexibility, plasticity and fractures balls, bubbles and agglomerates
} Annie Luciani, Stéphane Jimenez, Olivier Raoult, Claude Cadoz, Jean-Loup Florens

\section{- To cite this version:}

Annie Luciani, Stéphane Jimenez, Olivier Raoult, Claude Cadoz, Jean-Loup Florens. An unified view of multitude behavior, flexibility, plasticity and fractures balls, bubbles and agglomerates. Conference of the International Federation for Information Processing, 1991, Tokyo, Japan. pp.55-74. hal00910589

\section{HAL Id: hal-00910589 \\ https://hal.science/hal-00910589}

Submitted on 22 May 2014

HAL is a multi-disciplinary open access archive for the deposit and dissemination of scientific research documents, whether they are published or not. The documents may come from teaching and research institutions in France or abroad, or from public or private research centers.
L'archive ouverte pluridisciplinaire HAL, est destinée au dépôt et à la diffusion de documents scientifiques de niveau recherche, publiés ou non, émanant des établissements d'enseignement et de recherche français ou étrangers, des laboratoires publics ou privés. 


\title{
An Unified View of Multitude Behavior, Flexibility, Plasticity and Fractures Balls, Bubbles and Agglomerates
}

\author{
A. Luciani, S. Jimenez, O. Raoult, C. Cadoz, and J.L. Florens
}

\section{ACROE-LIFIA}

\begin{abstract}
The work presented here, is a part of a "modeler-simulator", capable of representing and simulating a large variety of physical objects: The Cordis-Anima system.

Based on particle physics, atomic interactions and the decomposition by network of lumped physical components, the Cordis-Anima system enables modelisation and real time simulation of a large variety of objects and scenes, rigid or deformable with collisions, fractures, sticking ...

However, the atomic representation of the objects becomes more unwieldy if there is more discontinuous behavior or if the desired resolution in the shapes or deformations are greater. We have added to the previous principles, the three following points :

$\checkmark$ "physical ball-meshing" which consists in an adaptative structural physical decomposition of matter.

$\checkmark$ "agglomerate-compacting" which consists in regrouping severals balls by one interaction law.

$\checkmark$ "physical-shaping" : the contour is seen as a physical interaction frontier between several agglomerates.

This provides access to a more macroscopic modelisation of large free form objects, or of objects whose structure is under dynamic modification.
\end{abstract}

Key words :

Computer animation - Physical models - physical interactions - Real time simulation - Physical shaping.

\section{INTRODUCTION TO PHYSICAL MODELING}

During its initial phase of evolution, image synthesis has gone from pixel manipulation to 3D modelisation. Whatever the importance of this leap forward, in terms of principles as well as the machines that materialise them, the breakthrough nonetheless was not sufficient to tackie the problem of animation adequately. Mathematical or geometric models, for the most part mainly directed towards indeformable objects - be they CAD models like CSG (Constructive Solid Geometry) and BR (Boundary Representation) or modelisation of natural objects using different interpolation functions (varieties of splines, Coons or Beziers surfaces) - are extremely unwieldy if used to describe high variability movements, the extreme case being highly deformable objects such as fluids or pastes.

Over the last two years, image synthesis has jumped another major hurdle, which is the introduction, in the object model, of behavioural properties. Some of these, such as the Reynolds multitude behaviour models [Reyn 87] or the Reeves "particle systems" [Ree 83] are formalisms that enable 
explicit cinematic restitution of the phenomena that may, or may not (e.g. anticipation phenomena) result from physical systems.

Others have tried to restitute the dynamics of physical systems by simulation, or what we refer to as "physical models". It is therefore clear that physical modeling can be considered as one sector of behavorial modeling. Indeed, from a conceptual standpoint it displays the same major advantages :

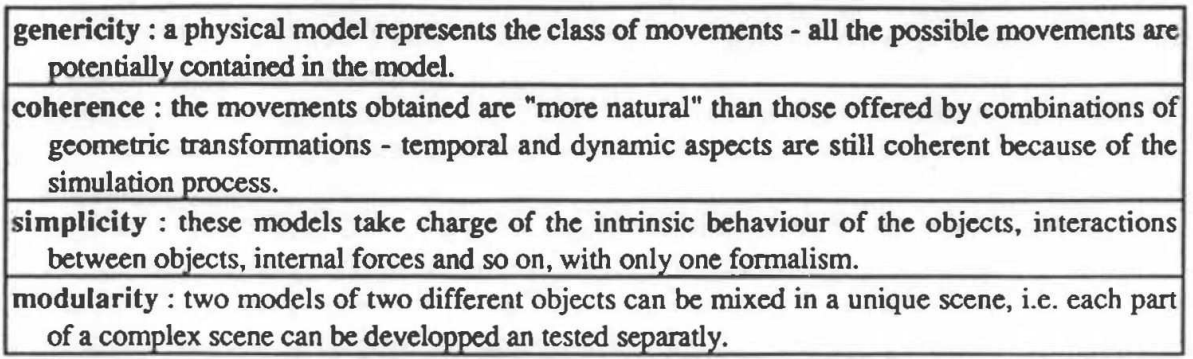

However there are distinct differences and these are to be found in the field of representation and in the algorithms. Non-physical models are based on systems of rules - we might even add on systems of arbitrary non limited behavorial rules. In physical modeling there is a canonical (in a mathematical sense) base of rules composed of physical rules. Variables are strictly state variables. These can be extensive variables (such as position, speed or deformation) and intensive variables (such as forces or constraints).Physical interactions cannot include anticipations such as actions to avoid obstacles or anticipatory behaviour proper to living beings.

From a simulation standpoint, we solve a set of great number of diferential equations and the algorithmic models are time implicit.

Physical modeling and simulation appeared in 1979 with the first developement of the Cordis-Anima system [Cad 79,81 - Luc 81]. Cordis-Anima is a system based on physical modeling for producing sound and/or animated images. Some time later, physical calculus was used at Ohio State University as a complement to other animation methods [Sch 84].

A great deal of research results have begun to accumulate in this field [Luc 84,85 - Cad 84 - Flo 86 Ter 87,88 - Bar 88 - Mil 88 - Hah 88 - Am 88 - DAH 89 - Flo 89 - Jim 89] since 1984. The net result is that the idea and the interest of physical models in synthesising a moving image is today acknowledged and has unanimously proved its initial worth.

In terms of physics, there are several computer representations available, and this obviously depends on the aim of the application. For example,

models and their implementation can be very different if we use computers to predict the behaviour of a physical system in as precise terms as possible (i.e. and application in numerical calculation) or if we want to use the computer as a modelor-simulator for physical systems and animation.

The Cordis-Anima system and the work presented in this paper have opted for the second goal. It follows that the modularity and the constructability of the models, in addition to real time in simulation, are major constraints in the computer representation of the physics.

The first part of this paper is devoted to the formal aspect in a computer physical modeler-simulator. The second part deals with the major optimisation needs occasioned by the constraints of real-time and of complex object simulation. 


\section{THE CORDIS-ANIMA FORMALISM}

It is pertinent to consider that a physical model of a physical object is itself a physical object, that is, it moves and is deformed when subjected to physical actions.

To achieve our objective of a modeler-simulator, we can therefore consider that such an object may be symbolised by a dipole, or more generally by an n-poles system, which associates the two sets of dual variables, extensives and intensives : $O(P, F)=F$

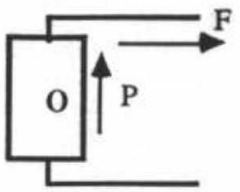

figure 1 : a physical object

It follows that all objects or sets of objects can be built by interconnected $n$-pole blocks which are in series, parallel, or in parallel/series.

This formalism conveys the structural discretisation needed in a modeler-simulator.

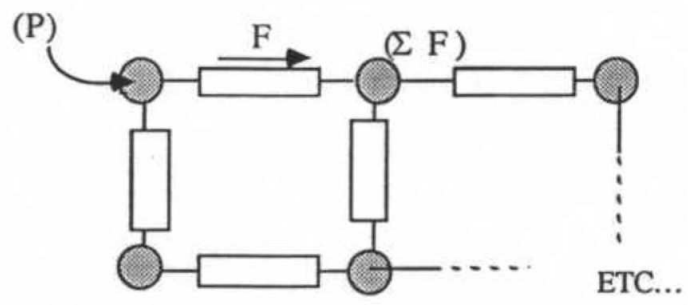

figure 2 : structural discretisation of physical object

This approach forces us to explicitly choose what each block contains, which is, in fact, the question of the minimal matter element and the underlying basis to interactions.

Physics, and the various chapters of mechanics offer many ways to define the minimal material element and to select and associate these two variables. The choice depends on the phenomena that interest us. This implies an option on a basic discretisation and modelisation principle for the real universe. However, the modularity constraint (cf. I) means that the expression of these laws should be the same on all levels of object description. These laws must be applicable to any network of the above type, even if this network represents only a part of an object, a complex set of objects or the entire scene. 
A usual choice (but not our own) is the "infinitesimal solid". The two dual variables are constraints and deformations. The integration of the behavorial laws of infinitesimal solids provide a macroscopic solid behavior.

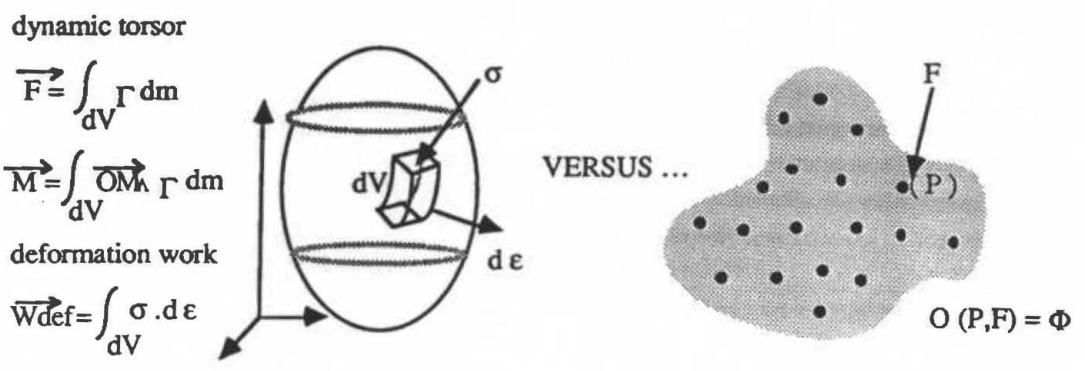

figure 3 : infinitesimal solid versus punctual representation of matter

The Cordis-Anima system, presented below, has opted for a "particle physics". The minimal element of matter is a punctual mass. Dual variables are positions and centered forces. Interactions links several of these elements of matter to make up an object or a scene.

Physical particles have mass and receive forces. They can physically interact and be submitted to gravity or all other kinds of forces.

In other "particle models", [Ree 83] for example, the particle systems have been chosen to simulate complex systems with a very high number of simple elements which are not necessarily supposed to be physical elements.

Cordis - Anima [Cad 81, Luc 84, Cad 84, Flo 89] has adopted the 3 following axioms as modelisation principles of physical space.

1 - Modelisation of matter in punctual masses : the minimal matter element is the punctual mass. It is represented by a discrete component, named the "matter component", whose physical parameter is the mass value. The calculation describing the behaviour of the component is provided by the fundamental dynamics equation Force $=$ Mass*Acceleration, expressed in terms of "finite differences". This calculation is oriented to provide the mass position from the forces that are applied to it. This component therefore presents itself as an oriented dipole [force $\rightarrow$ position].

2 - Modelisation of the continuous behavior of the material : the material, that is normally defined by parameters of elasticity, viscosity, or others is represented by discrete components without mass, named the "material component", whose characteristics are elasticities or viscosities, considered under their continuous aspects, with neither thresholds nor hysteresis. The calculations are provided by the basic equations describing continuous behaviour $\mathrm{F}=-\mathrm{K} * \mathrm{D}$ (the force is proportional to the distance) and $\mathrm{F}=-\mathrm{Z}^{*} \mathrm{~V}$ (the force is proportional to the relative speed). These calculations are oriented to provide forces from the positions. These components present themselves as criented quadripoles [two positions - $>$ two forces].

3 - Modelisation of the structure of matter and of objects : the "material components" that enable modelisation of the material are equipped with a double logical function. The first is to "link up" the components representing the punctual masses. The second is to carry out this liaison 
according to a transition status logic that enables the parameters of the material to be modified in function of conditions affecting the parameters or variables. This enables homogeneous access to non-linear behaviour, continuous or discontinuous behaviour without any addition of basic primitives. Collisions, non-linear parameter modifications, such as elastic behaviour over large displacements, all non continuous behaviour, with or without hysteresis (like plasticity) can thus be processed by this unique formalism.

There are thus no specific collision or fracture algorithms for rigid and deformable objects. This choice of modelisation enables us to modelise the microscopic structure of matter of an object in the same way as the non-linear macrostructure of a scene. It clearly evidences the proximity between phenomena situated on different levels - an elastic collision, a rupture (exceeding the elasticity threshold), or plasticity for example.

The fact that the components can be represented in terms of quadripoles and dipoles enables us to define simple connexions between components. The representation of physical objects by a network of interconnected boxes is particularly adapted to model construction modularity. The extremely restricted number of base algorithms lend themselves well to vectorisation.

The network which describes an object or a set of objects in physical interaction can be simulated component by component, and this is a great advantage as regards the model experimentation, depending on the modular design.
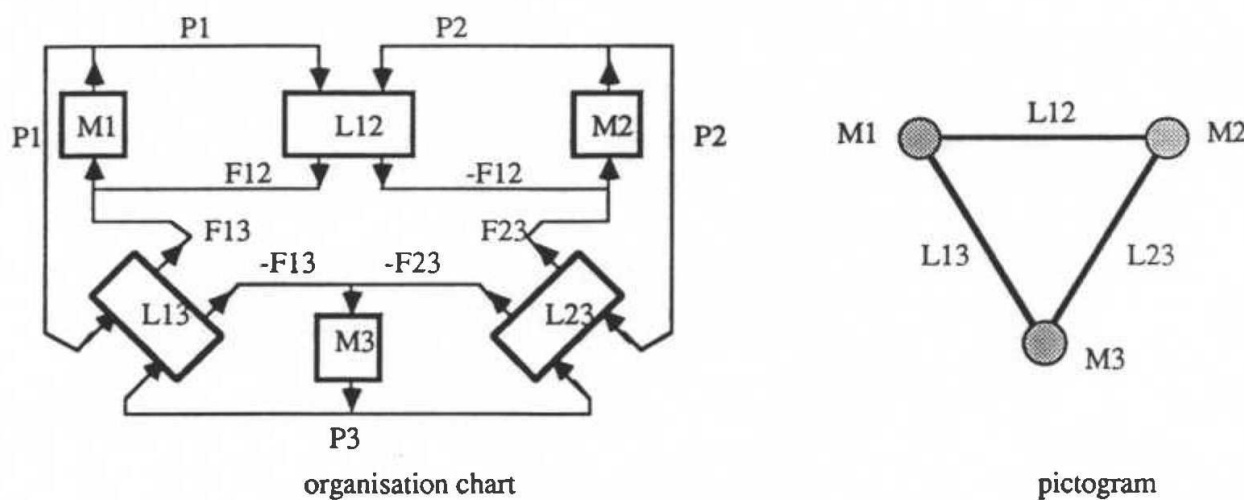

organisation chart

pictogram

figure 4 : an example of a Cordis-Anima model

Major consequences arise from these principles :

- The interaction between the human operator and the simulated objects is similar to the interactions between physical objects.

- The simulated physical objects are physically manipulable.

- By using gestual transducers, a user can apply forces, or positions to the object. This object responds by movement and deformation.

- Such an object is perceptible in a proprio-tactilo-kinesthetic manner. By using force feeback transducers, the user can actually feel forces and displacements. 
There is hence no control problem. The gestual bandwith is large enough to convey expressive manipulations. This formalism is more general and therefore enables modelisation and simulation of all types of objects, be they rigid or deformable. This equally applies to objects linked by any type of interaction, linear or otherwise.

In contrast, this kind of atomic description and simulation of a scene becomes more unwieldy if there are:

- more complex discontinuous behaviour events (for example, dry friction between a track vehicule and a non-homogeneous ground).

- a great number of masses and interactions as for example in the agglutination of finely granulated bodies (powders, fluids, ....).

Because of the above, two more specific optimisations have been developed [Jim 89] as a special supplement to the Cordis-Anima modeler-simulator. They are totally compatible with the Cordis-Anima formalism but they provide access to a more macroscopic modeling of free form physical objects or of objects and scenes whose structure is under frequent modification.

These are suitable when there are :

- a great number of the same elements (homogeneous materials for example).

- or in the case of non-linear interactions, when the non-linearities depend only on a condition over one variable (collisions for example).

Depending on the importance of these case, these optimisations can be seen as another modeling principle, which we have named "agglomerate with univariable non-linear interactions".

Nonetheless, as a part of the Cordis-Anima modeler-simulator, they fulfil the general conditions of modularity; they do not restrict the domain of representation and they can use the force feedback interaction devices for physical real time interactions with the operator.

\section{AGGLOMERATES WITH UNIVARIABLE NON- LINEAR INTERACTIONS.}

The context is one of buildable, manipulable, and interactional physical objects. The basic principles remain the same as those described and discussed above - the choice of particle dynamics in opposition to solid dynamics; the use of interaction between particles to define complex objects or set of objects ; the modelisation by network of lumped physical components.

\section{III.1 Balls, bubbles and agglomerates}

The fundamental change brought in, compared to the previously described Cordis-Anima formalism, resides in a new way of breaking down a piece of matter. The principled is rooted in the construction of aggiomerates, that are pieces of matter which present themselves as a milieu. The minimal matter element here is the "physical ball".

We replace the Cordis-Anima physical points, nominatively coupled to another physical point, by composite entities named "balls". A ball is never alone. What we designate as "balls" are a group of 
punctual masses that come into interaction amongst themselves via a distributed interaction function. The prime parameter of this function is the interaction zone.

Each ball is a punctual mass plus a spherical non-entrance zone (impenetrability). In the case of a non-exit zone, we refer to "bubbles" (figures 5). This zone appears only when interaction occurs. A set of scattered balls is merely a group of punctual masses .

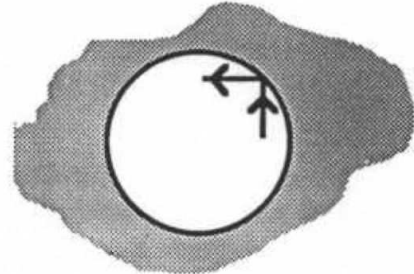

Bubbles

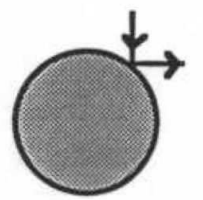

Balls

figure 5 : Bubbles and Balls

Thus the initial principles have been specialized with "physical ball-meshing" as an adaptive structural decomposition of matter. A piece of physical matter will be presented as a set of different sized physical balls.

We have also introduced "agglomerate-compacting". An agglomerate is a group of balls plus a law of interaction between each balls of the set. This law can be attractive, repulsive or more complex (see below).

These two concepts could be written as :

$$
\begin{array}{lll}
\text { mass }+ \text { interaction zone } & = & \text { ball } \\
\text { balls }+ \text { interaction law } & = & \text { agglomerate }
\end{array}
$$

As a consequence, we can now speak of "physical-shaping". The contour of an object can be defined as the physical interaction frontier between several agglomerates.
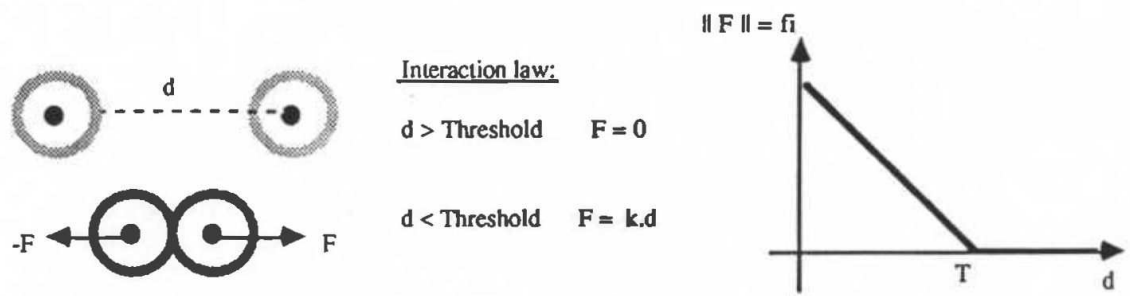

figure 6 : balls under interaction - the elementary law of interaction

As we said above, agglomerates are composed of a group or set of balls linked together by a specifically unique interaction law. The overall interaction law should be observed as an intrinsic property of the agglomerate.

A $N^{*}(N-1) / 2$ liaison module calculates the mutual interactions between the masses (figure 7). 


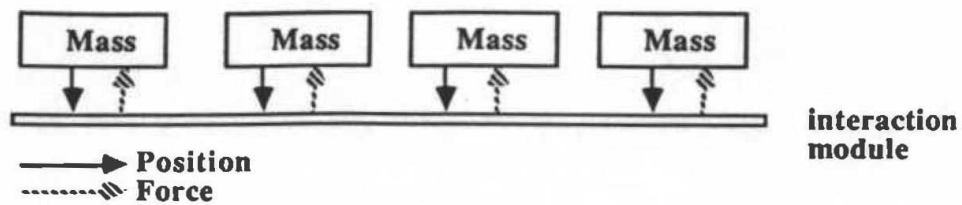

figure 7 : Interaction Module

A particular, and moreover interesting case, is the intermolecular law of liaison (Van der Waals). It enables us to build consistent agglomerates with a centred shape. However, other types of laws are possible.

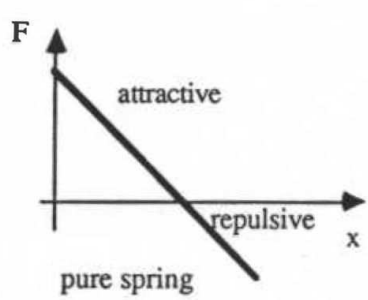

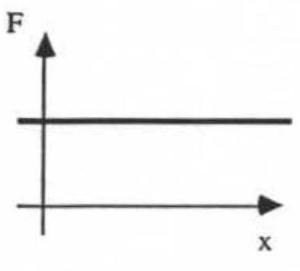

Gravity

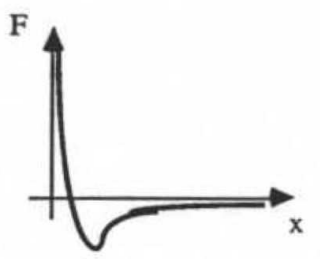

Intermolecular interaction

figure 8 : interaction functions

In the case of the agglomerate, the output of the interaction functions depends on only one variable and then, the interaction function can be represented by piecewise linear function (as shown in figure 6 and 8). It is easy to define a generic algorithm to design piecewise linear functions. In addition, because of the formalism of punctual representation, the forces are summed on masses, and thus we can put several interactions in parallel and obtain complex interaction functions from simple linear piecewise functions. Because of this approach, we do not need to have more than two or three pieces in the interaction primitives.

This kind of interaction is an important case of the "conditional link sub-system" implemented in the Cordis-Anima system [Cad 81, Luc 84, Luc 90(1), Luc90(2)] which allows us to describe and simulate general non-linear interactions from "fusible links" as in [Ter 89] to general non-linear multivariable interactions as a bowed string, snow/ski interactions, or track vehicule/non-homogeneous ground interactions, where complex temporal sequences of states occur.

\section{III.2 Agglomerate properties}

An agglomerate is an accumulation of undifferentiated balls with a global shape which results from a dynamic process.

The shape does not pre-exist, it is achieved.

It is one state of equilibrium. These states of equilibrium are the peculiar points of a generator process. One characteristic of these particular points is the degree of stability. Thus, an agglomerate can intrinsically break up, without any addition of extra information. A mass violently striking an agglomerate stabilised by Van der Waals, or by gravity, can break it. The agglomerate may not necessarily be able to totally reconstitute itself. It is also possible to define sticky agglomerates so that they cannot be broken apart. 
The interaction function and the number of balls define the variety of agglomerate shapes. However, the intemal arrangement of the balls depends on dynamic conditions given to the generator process. Two balls could be permuted without any change in the global shape.

In contrast to Condis-Anima, the position of each ball is not determined when the model is constructed (balls were dropped in quasi-random positions). In fact, it would be useless. Thus, the given model is not really a model of the final object, but an object-making model.The underlying thought process to modelisation work, is a thought mode of movement and temporal processes.

\section{III.3 Objects}

An agglomerate is an elementary homogeneous object. In the same way as an agglomerate is a set of balls under interaction, so a complex object can be described by a set of interacting agglomerates. Each couple of agglomerates could be linked by its own interaction law.

$$
\begin{array}{llll}
\text { agglomerateS } & +\mathrm{P} \text { interaction lawS } & = & \text { object } \\
\text { objectS } & +\mathrm{Q} \text { interaction lawS } & = & \text { scene }
\end{array}
$$

To calculate all the interactions between all the balls of two agglomerates, we use an optimized "NxM liaison module". This module corresponds to a unique interaction law distributed over the NxM couples of the cartesian product of two agglomerates.

Unlike the agglomerate, these can be linked together by several different sorts of interaction functions in parallel. It is therefore sufficient to define as many "liaison modules" as required, since the summation of the forces is effected in the balls of the agglomerates.

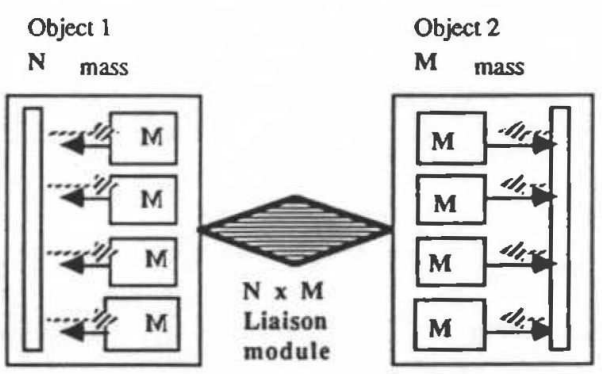

figure 9: NxM Liaison Module

\section{III.4 A proposition for the construction of malleable objects}

The following structure is an operational guide to modelise (more or less rigid) solids by the "physical ball composition" approach. An object can be structured in three relative layers, that are not all necessarily present :

- A deep layer or "nucleus" : this represents a centering function that the object organises itself around. The nucleus is either undeformable or only slightly deformable. In the simplest case, it may be reduced to a large attractive or repulsive ball, and is thus indeformable. But this nucleus 
can also be composed of several masses and springs to define, for example, a cube or any other sort of structured object.

- A derm : this will bear the most substantial deformability qualities. Its thickness represents the depth and area of the deformation quite diroctly.

- An epiderm, or "skin" : its role is to reinforce the agglomerate's cohesion. This is where the notion of "surface tension" will be localised.

Each layer is characterised by a specific interaction function between its constituents, that are in complementarity to their neighbouring layers. The object interaction with the exterior varies more or less in depth, depending on the layers concerned.

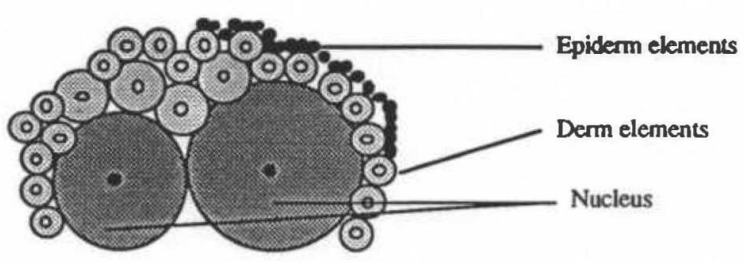

figure 10 : structured object

The main role of this organisation is to structure the interactions between objects, and consequently, to lower their number. For example, in the case where a skin layer exists, it handles the interactions with the outside. In the more extreme case of a scene composed of many objects which can shatter into fragments, and then fall again into new objects, all the layers, including nucleii, must be affected by the outside interactions.

\section{III.5 Simulation examples}

\section{A multitude of particles in interaction}

The water of the cascade (see photograph 1) is modeled as a set of indeformable particles in nonpermanent elasto-viscous collision interactions. A big difference between this model and other "particles sytems", is the possibility to obtain pool of water, whirlpool and turbulence - which means that each ball could interact with all the others. This type of effect cannot be obtained with a simple superimposition of independent trajectories.

The principle is similar in the next example : a ball thuds into an heap. Here again, effective interactions are necessary.

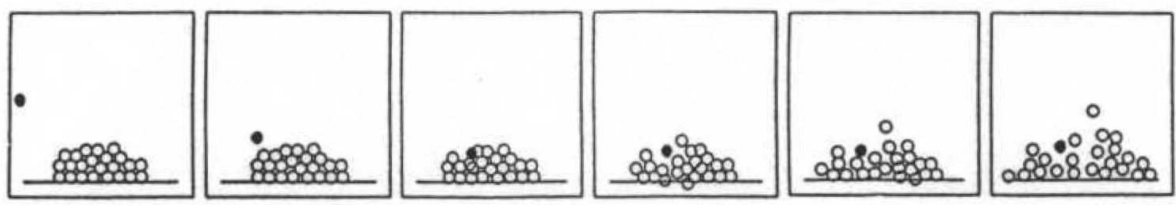

chronogramm 1 


\section{A simple structured object : the rubber ball}

This is a stable, spherical, and slightly deformable object. It is structured in two layers. A nucleus confers the overall shape to the object ( a 3D ball or a 2D disc). The derm is composed of a set of interrepulsive balls. The nucleus/derm interaction law is "intermolecular". The derm balls take up a position of equilbrium by positioning themselves regularly around the nucleus and form a regular crown. When the object runs into a wall, it is slightly crushed, and the derm balls that are in contact with the wall are separated. When the object moves away from the wall, it recovers its initial spherical shape. This model introduces the notions of adaptive meshing and of deformation locality (see photograph 3 ).

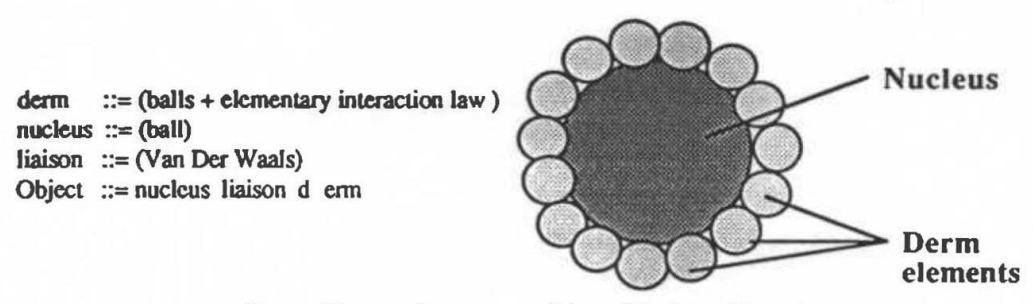

figure 11 : an elementary object (Nucleus-Derm)

\section{The rubber ball - separation and then regrouping}

This example shows how the previous deformable object is equally transformable without any addition of other functions. Cases of non predefined splitting or of "re-sticking" are entirely taken into consideration. The shape of these objects is not defined as such, but it is made up. It is the free expression of dynamic forces that lead to stable forms. This also illustrates that the first level of complexity, - i.e. the dynamic modification of the structure in the object - is allowed for in the agglomerate. The object can shatter into elementary fragments.

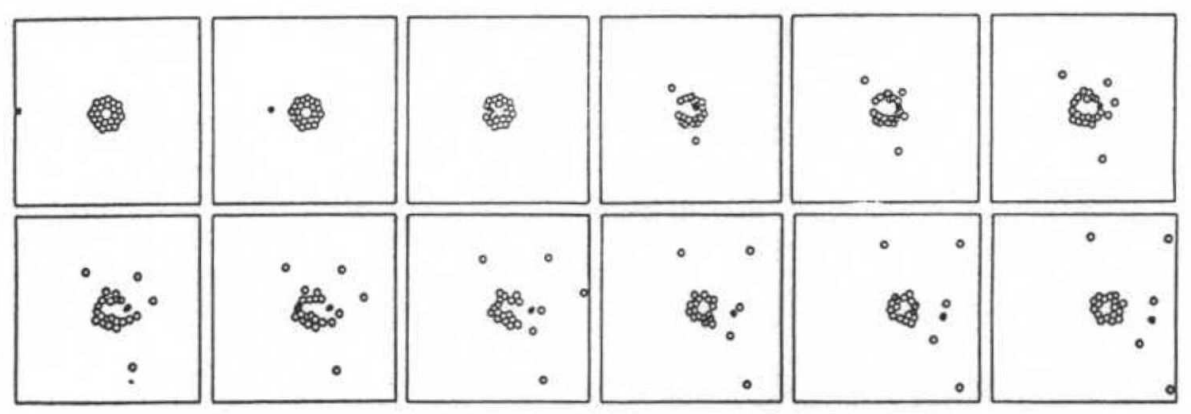

chronogramm 2

4. The rubber ball - sliding without rolling, rolling without sliding

During the contact with a flat floor - that is formod by little adjacent balls - the rubber ball may present a sliding movement without rolling. It occurs when the size of the floor balls is smaller than those of the derm, or when the floor is locally extra flat (simulated by a very large ball).

If the derm of the rubber ball is very thick and the nucleus very small, it has a large depth of deformation. Placed on a flight of stairs - that is obtained also by "ball-meshing" (figure 12) - it tears down the stairs, hugging the steps' shape. It rolls, but it does not slide (see photograph 4). 


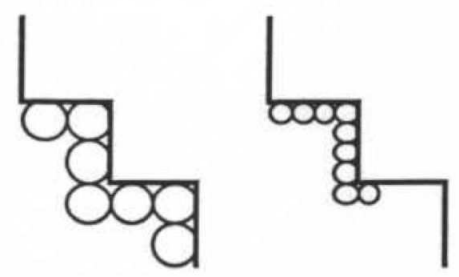

figure 12 : "ball-meshing" stairs

\section{Mouldable object}

The photograph 5 show a piece of plasticine which is mouldable by the operator in real time. The model of this plastic object is obtained by an agglomerate composed about 30 balls linked by a repulsiveattractive interaction function. The attractive zone is chosen to be lower than a 2 ball radius. The object has the shape that the operator gives it. The plasticine can broken up and then re-stuck together according to the manipulation. We can easily knead the paste, tear and recompact it, and this in real time.

\section{A file model example}

The model below describes a sor of rattle composed of a tetrahedron enclosed inside a hollow sphere. The sphere can rebound on a flat floor and the tetrahedron inside the sphere. This model is not a program but just the description of elementary objects, and interaction laws between them. In this simple example, movements merely result from gravity and intemal interactions effects - five lines more were needed to enable real-time manipulation with force-feedback gestual transducers.

Each line is composed of a keyword, an identification name (if needed) and a set of parameters. It should be noted that "DEFMASS" (as DEFFI) is just used to associate name to numerical values whereas "DEFMOB" is used to allocate $\mathrm{N}$ balls descriptors, to specificy their mass (named), the topology of the internal interaction law (AGGLOmerate), plus the interaction law itself (named). For example, "DEFMOB foo $10 \mathrm{n}$-m AGGLO $n \_i n t l "$ means : allocate an agglomerate descriptor named "foo", composed of 10 balls (each ball fool, foo $2, .$. has a mass " $n$-m") with an interaction law named "n_intl".

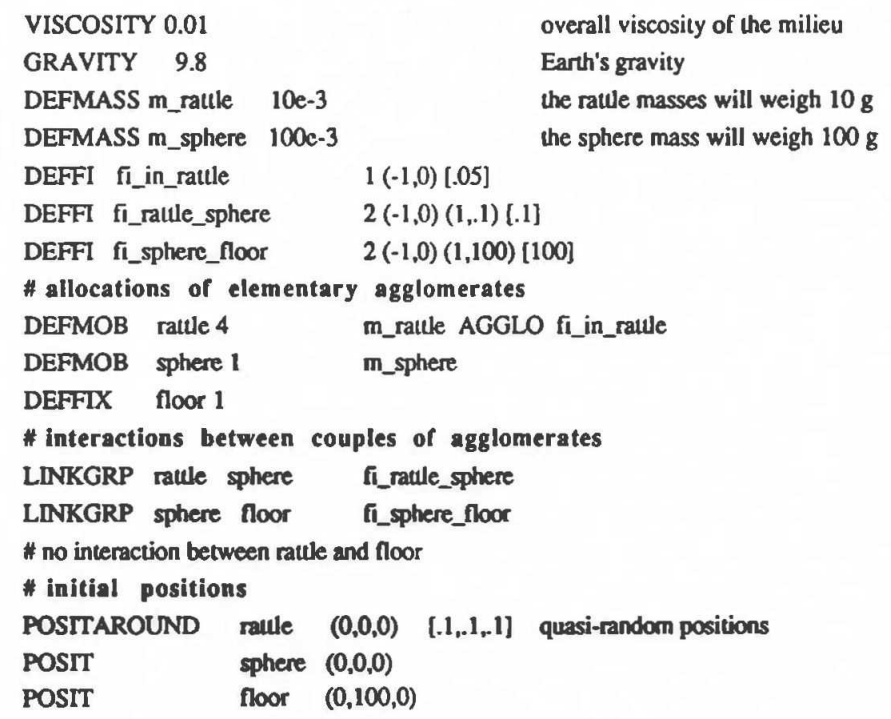


The initial positions of the material points of the rattle are selected in random fashion. Four material points linked together by springs of a given length $(5 \mathrm{~cm}$, see fi_in_rattle) will spontaneously organise themselves into a tetrahedral shape, whatever their initial configuration. Moreover, the sphere will "swallow" or absorb the tetrahedron even if the latter is initially on the outside. The initial status of the rattle is not determinant here since only one stable structure exists (the rattle masses organised into a tetrahedral, the tetrahedron inside the sphere, and the sphere above the floor)

The ground is a hollow sphere with a $100 \mathrm{~m}$ radius. Its center is fixed rigidly at an altitude of $100 \mathrm{~m}$. It therefore behaves like a quasi-flat floor surface. We often use such methods to reduce the effective number of balls ; in some case, ball-meshing can be avoided.

\section{III.6 Optimisation, parallelisation, vectorisation}

\section{Physical meshing}

Our decomposition of physical matter is : adaptive, punctual and isotropic. It therefore offers a considerable gain in calculations compared to the more classical approaches that employ geometric meshing of solids.

Physical Meshing is adaptive : there is no unique best model for a given object. Structural variations could be used exactly as parameter adjustments to obtain very precise effects. The meshing accommodates the shape specificities of the object as well as the deformation-transformation locality (figure 13).
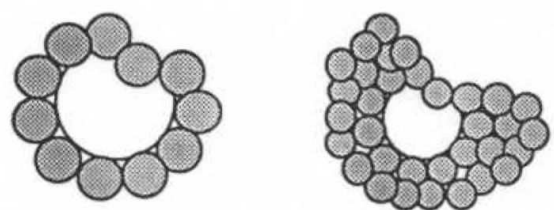

figure 13: Two meshing examples

Physical Meshing is punctual : as the calculations are based on particle physics they never bring in rotations, projections, vectorial products and so on. The ball has only three degrees of liberty. Its calculation is linear. The equations are only a sum of products. It is the same for each of the 3 degrees of liberty, and for all the balls of all the agglomerates. If the complete scene has $\mathrm{N}$ balls, we can easily vectorise the calculation of all these equations.

Physical Meshing is isotropic : there is no integration of any potential function.. The interaction functions are centered. The calculation only involves the distance (or relative speed), which is calculated once for each sampling step. These functions can be represented by linear piecewise functions. These may be expressed in algebraic form, with certain approximations.

We shall only provide two major examples to illustrate the efficiency of this calculation : the first is an example of interaction calculation (intermolecular) ; the second shows how an elementary solid is obtained from punctual masses. 


\section{Intermolecular interaction calculation}

Physics proposes a potential energy model (Lennard-Iones model) which enables definition of a potential family expressed as : $V n=(S / d) 2 n-2 *(S / d) n$ where the threshold $S$ represents the zone of minimum potential. The force family derived from these potentials is written as :

$F n=-\operatorname{grad} V n=-(\partial V n / \partial x, \partial V n / \partial y, \partial V n / \partial z)$ which produces $F n=F i(d) * D p$

where $\mathrm{Dp}=(\mathrm{dx}, \mathrm{dy}, \mathrm{dz})$ is the "distance vector" of norm $\mathrm{d}$ the Fi functions are expressed in the form (figure 14) : Fi(d) $=2 * n^{*}\left(S^{2 n} / d^{2 n+2}-S^{n} / d^{n+2}\right)$

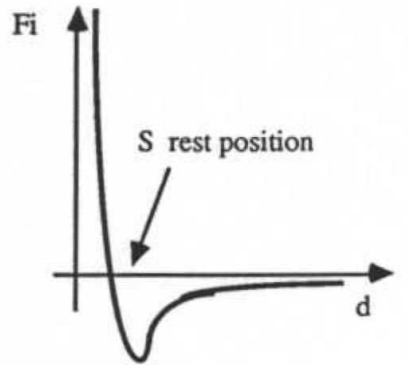

figure 14 : Lennard-Jones Interaction

Below is an example of this type of function approximated by a three piecewise linear function (fig.15) :

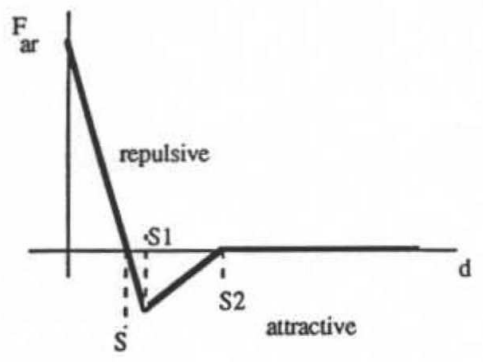

figure 15 : attractive/repulsive interaction fonction

Which, in the same way as any linear approximation, can be expressed as a sum of absolute linear term values ( here in three terms):

$\operatorname{Far}(d)=A_{0} *|d|+A_{2} *\left|d-S_{1}\right|+A_{3} *\left|d-S_{2}\right|+C$

where $A_{0}, A_{1}, A_{2}, C$ are real constants which depend on $S, S_{1}, S_{2}$.

Concerning the complexity of the interaction function - in this case, the number of pieces - we can make two remarks : in the major cases, three pieces are sufficient ; we can also increase this number by superimposing several functions in parallel. 


\section{Construction of an elementary solid (6 DOFs) by ball composition}

A primary challenge for this type of modelisation is to be able to simulate a solid, with 6 degrees of freedom with the previous 3D components. Photograph 2 shows an elementary solid composed of 4 balls which form a tetrahedron, enclosed inside a rectangular compound, and stabilized by the interaction function described above. This object is stable, strikes the walls, bounce, and displaces itself in translation and in rotation after the impacts. The chronogram below shows a similar solid, which is flat and composed of 3 balls (a 4 DOF object composed of two 2 DOF balls).
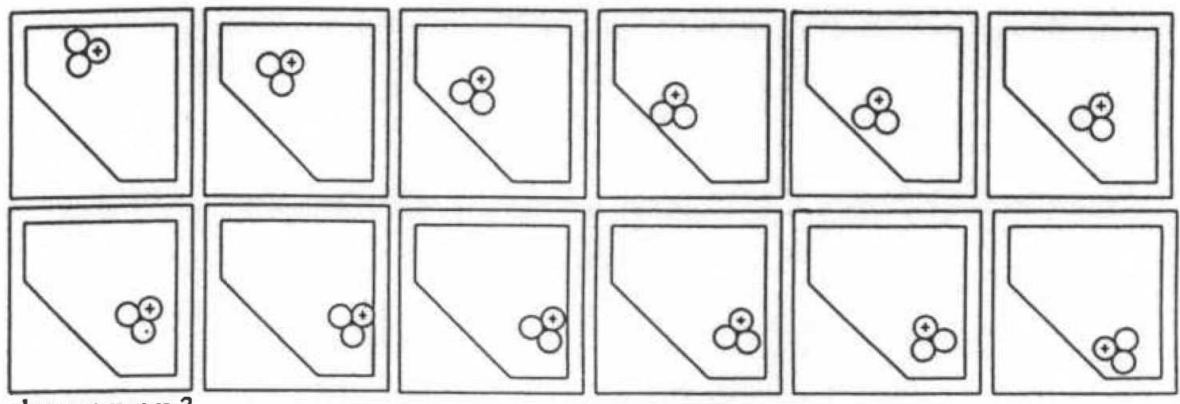

chronogramm 3
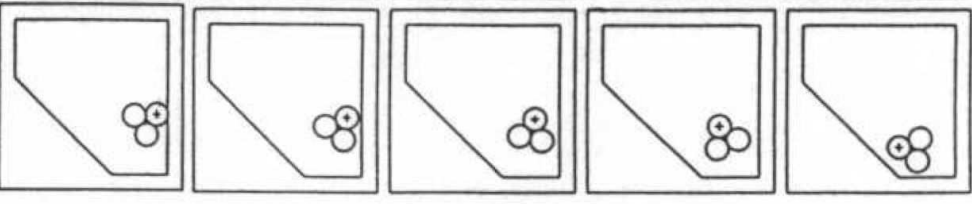

\section{THE CONTOUR}

"Contour" is a rich, multiform and complex notion, that cannot be reduced to the current methods in image synthesis (boundary representation : parametric surfaces and faceting). Our type of modelisation enables frequent or far-reaching structure modifications, so the generation of the contour demands thorough prior definition of the notion.

The contour is what establishes the unity of the object in its relation with the outside, and what implicitly distinguishes an interior (the object) from an exterior (the environment). It characterises the object as an individuated shape. It is a "membrane", "a threshold", and its materiality is sometimes highly partial, as, for example, in the case of a candle flame. It sustains the interactions with the exterior, and these interactions are of a very varied nature, which is why it is sometimes more the coherence between phenomena that allows us to establish the existence of a contour. In short, the object is seen, manipulated, and interacts through its contour.

When a model object includes an epiderm, there is no doubt that the latter conveys the outside shape of the object. Figure 16 gives an example of an epiderm model. The material skin, that is composed of visco-elastic Mass-Liaison bipole elements [Cad 79, Luc 81] plays the role of cohesion for the object like an elastic membrane, and because of this very fact (setting the border between the object and its environment) it is the contour. 


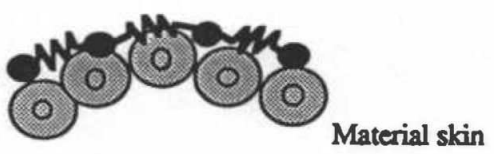

figure 16 : material skin

The next chronogramm shows an example of material skin.
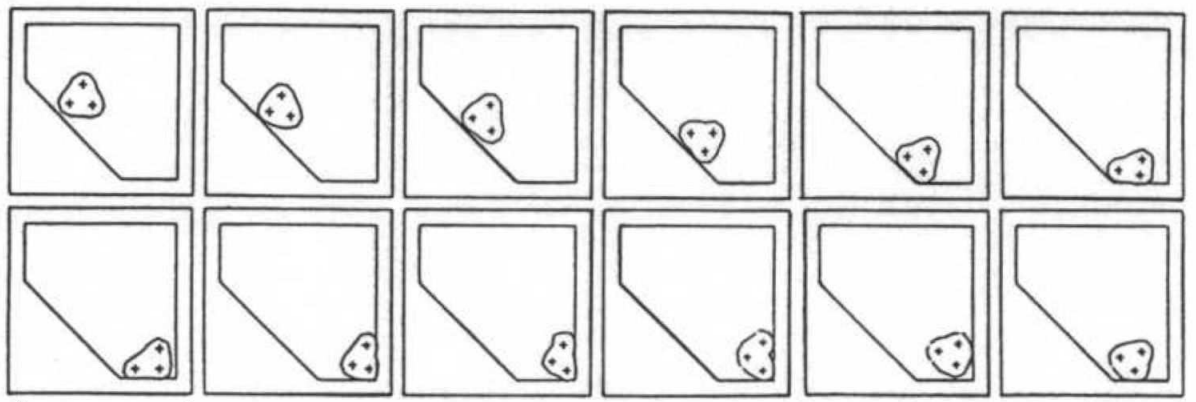

chronogramm 4

On occasions, objects have a weaker internal cohesion, and where the model does not require an "epiderm" layer. For example, this could be said of a more or less viscous fluid - such as a drop of oil or of mercury, or materials that are capable of being broken up or stuck back together - such as modelling clays. In this instance the contour can no longer be sustained by a structurally stable material skin. It should be noticed that in the commonest instance, the contour, when it exists, is a special feature, as a discontinuity or a zero-level, of the interaction function between the object and its environment.

Thus, for an agglomerate as we have just described, it is relatively easy to define a global interaction law, as the sum of the interactions which would be produced by each ball, in a point $x, y, z$ of the empty space. We can then determine the main discontinuities of this 3D interaction field. Finally, we must detect this frontier in order to display it.

For that, we might use systematic or adaptive scanning techniques but the cost of the operation is inacceptable for a simulation that has to take place at a minimum of $25 \mathrm{~Hz}$.

So, we have focussed on a technique that could be called "dynamic scanning". This means running a free mass with an initial speed over the object border. This mass is influenced by the global force field $\mathrm{Fp}$, derivated from the intertaction fied. The successive positions of the mass, defines an approximation of the object envelope (figure 17).

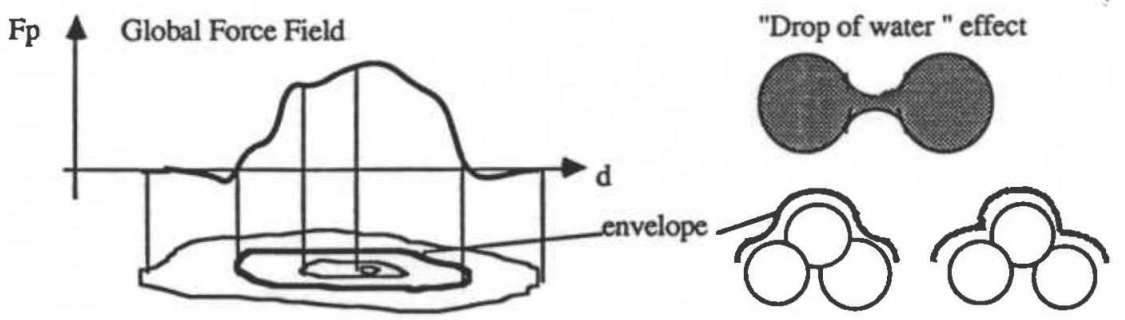

figure 17 : "dynamic scanning" 


\section{PHOTOGRAPHS}

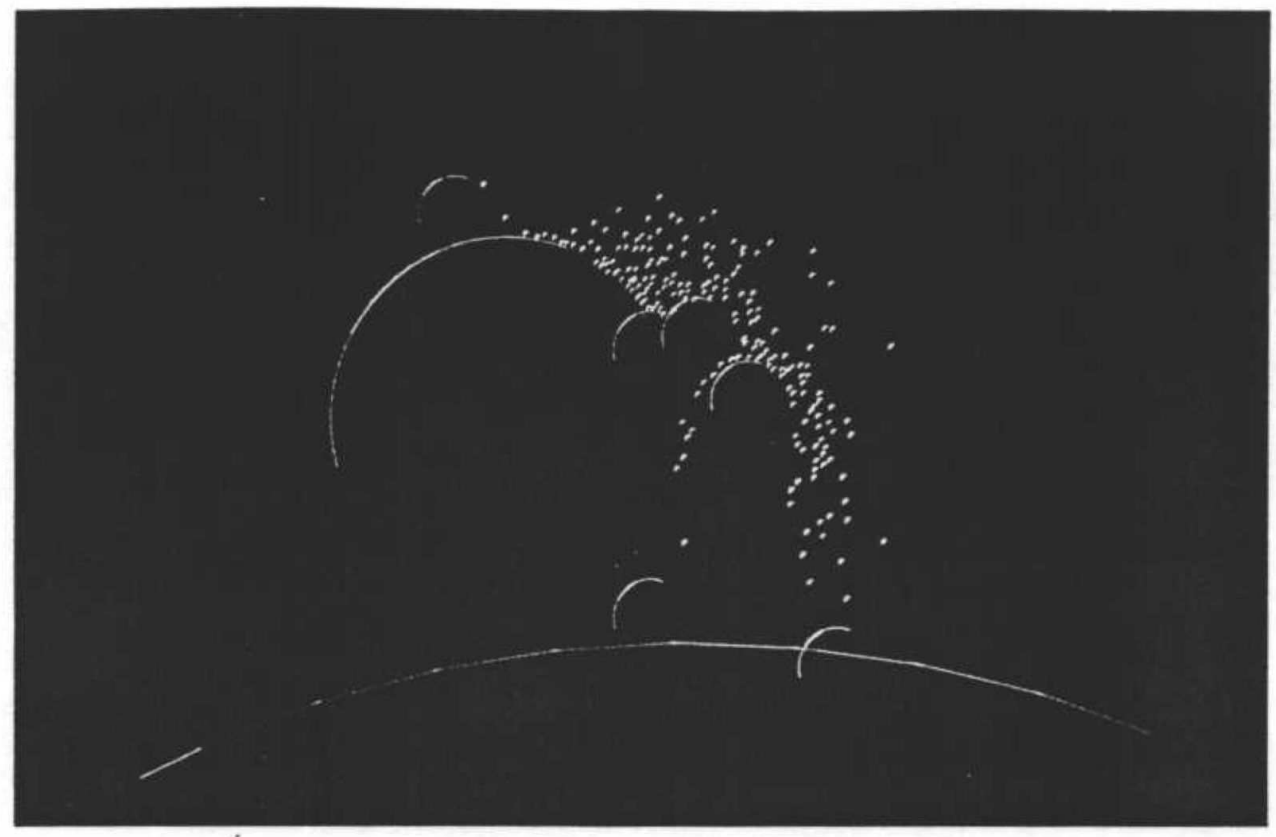

Photograph 1 : The Cascade 

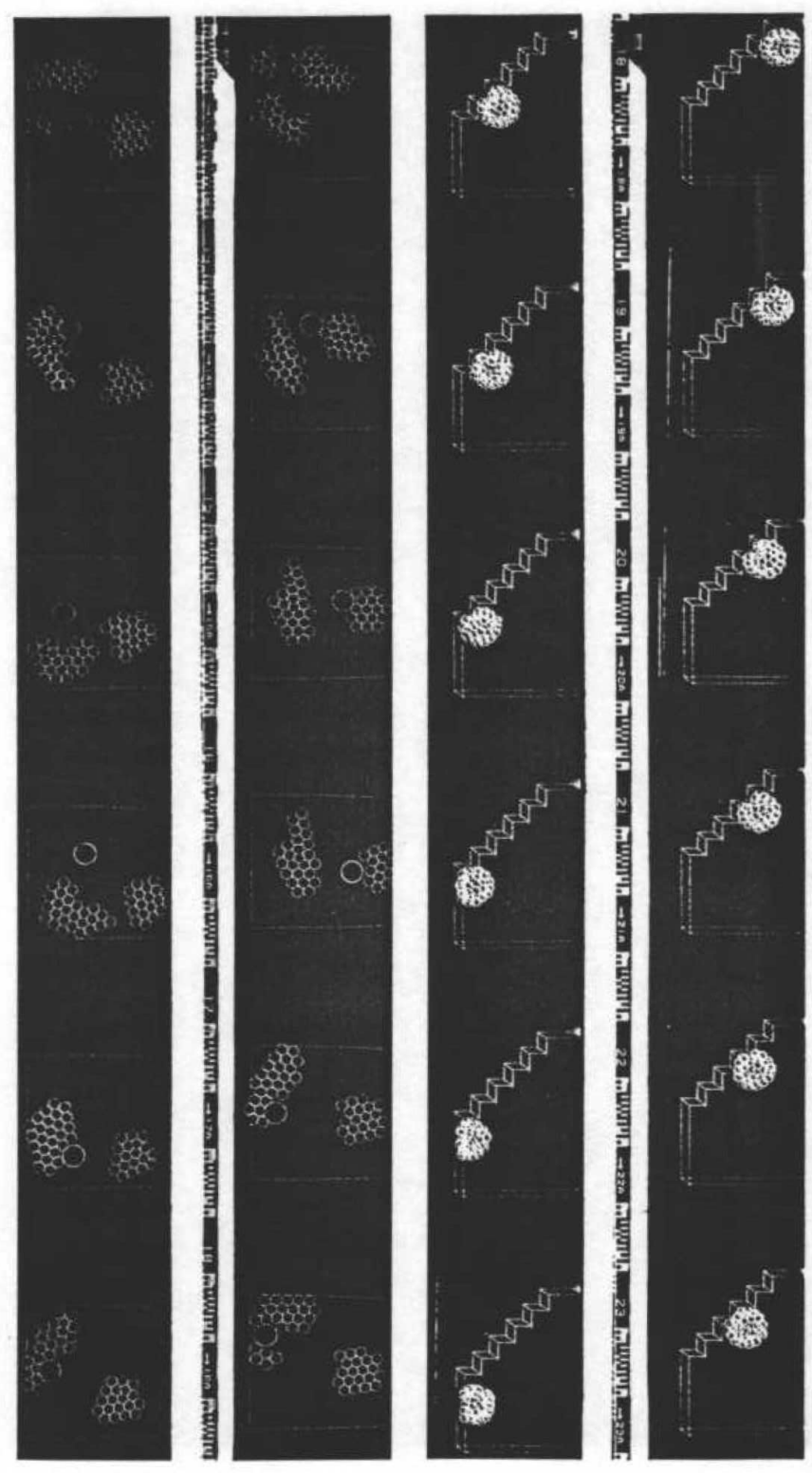

${ }^{20}$

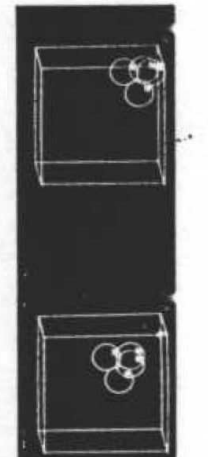

$\frac{692}{63}$

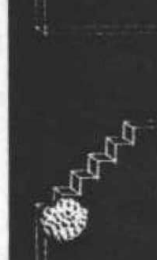

$+3=$

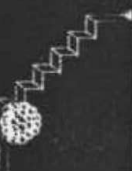

$\pi^{\pi^{4}}$

\%

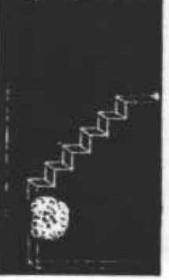

(1958

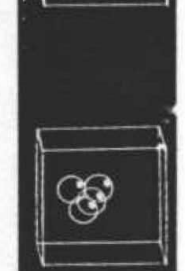

80

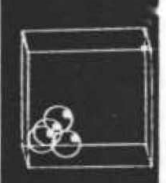

$\frac{8}{904}$

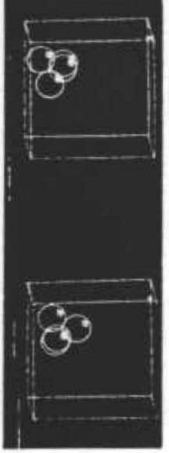

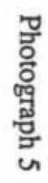

뭉

号

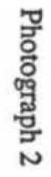




\section{REFERENCES :}

[Cad 79] C. CADOZ - " Synthèse sonore par simulation de mécanismes vibratoires" - Thèse Doctorat Electronique - INP - Grenoble - 1979

[Cad 81] C. CADOZ, J.L. FLORENS, A. LUCIANI - " Synthèse musicale par simulation de mécanismes instrumentaux" - Transducteurs gestuels retroactifs" - Revue d'acoustique N59 - 1981

[Luc 81] A. LUCIANI - "Anima, un outil de création d'images animées par modèles physiques" DEA Electronique - Grenoble - 1981

[Reev 83] W.T. REEVES - "Particle systems - A technique for modelling a class of fuzzy objects" SIGGRAPH 83 proc $359-376$

[Cad 84] C. CADOZ, A. LUCIANI, J.L. FLORENS -"Responsive input devices and sound synthesis by simulation of instrumental mechanisms : the Cordis system" - Computer Music Journal - N³ - 1984 - reprint in "Music Machine" - MIT Press

[Luc 84] A. LUCIANI - "Modélisation et animation gestuelle d'objets : le système Anima" - ler colloque Image Cesta - France - 1984

[Sch 84] M.K. SCHWEPPE - "Motion in computer Graphics" - 1er Colloque Image - Cesta - France $-1984$

[Luc 85] A. LUCIANI - " Un outil informatique d'images animées - modèle d'objets, langage, contrôle gestuel en temps réel" - Thèse Doctorat Informatique - INP - Grenoble - 1985

[Flo 86] J.L. FLORENS - " Optimized real time simulation of objects for musical synthesis and animated image synthesis" - Proceedings International Computer Music Conference - 1986

[Raz 86] A. RAZAFINDRAKOTO - "Langage d'objets pour l'animation, implantation temps réel" Thèse d'Université - Grenoble - 1986

[Reyn 87] C.W. REYNOLDS - "Flocks, Herds and Schools : A distributed behavorial model" SIGGRAPH 87 proc 25-34

[Ter 87] D. TERZOPOULOS - " Elastically Deformable Models" - Proceedings SIGGRAPH 1987

[Am 88] B. ARNALDI - "Conception d'un noyau d'un système d'animation tri-dimensionnelles intégrant les lois de la mécanique" - PhD thesis, Rennes I, juillet 88.

[Bar 88] R. BARZEL, A.H.BARR - "A modeling system based on dynamics constraints" Proceedings SIGGRAPH 1988

[Hah 88] J. K. HAHN - " Realistic animation of rigid body" - Proceedings SIGGRAPH 1988

[Mil 88] G. MILLER - "The motion dynamics of snakes and worms" - Proceedings SIGGRAPH 1988 
[Pla 88] J.C. PLATT, A.H. BARR - "Constraint methods for flexible models" - Proceedings SIGGRAPH 1988

[Ter 88] D. TERZOPOULOS - " Modeling inelastic deformation" - Proceedings SIGGRAPH 1988

[Cad 89] C. CADOZ, J.L. FLORENS - "Le modèle Physique - Référence et Artifice dans la création musicale par ordinateur", in - MIT Press - 1989 - à paraitre

[DAH 89] G. DUMONT, B. ARNALDI, G. HEGRON - Mechanics of solids for computer animation" - Proc. Pixim 89 p.293-308 - Paris - octobre 89.

[Flo 89] J.L. FLORENS, C. CADOZ - "Le Modèle Physique - Simulation de l'univers instrumental" - in " - MTT Press - 1989 - à paraitre

[Gas 89] M.P. GASCUEL - OSEA - "Un nouveau modèle de matière pour traiter les collisions entre objets déformables" - Pixim 89.

[GV 89] M.P. GASCUEL, A. VERROUST - "Animation à l'aide de la dynamique : état de l'art" Rapport LIENS - ENS Paris - Mai 89.

[Jim 89] S. JIMENEZ - "Modelisation et simulation d'objets volumiques déformables complexes" DEA Informatique - INP - Grenoble - 1989

[Ter 89] D. TERZOPOULOS, J PLATT, A. BARR, and K. FLEISCHER - " Heating and melting deformable models (from goop to glop)." - Proceedings SIGGRAPH 1988

[Dum 90] G. DUMONT - "Animation de scènes tri-dimensionnelles : la mécanique des solides comme modèle de synthèse du mouvement" - PhD thesis, Rennes I, mai 90.

[GVP 90] M.P. GASCUEL, A. VERROUST, C. PUECH - "Animation with collisions of deformable articulated bodies" - In EUROGRAPHICS Workshop on Animation and Simulation September 90 .

[Gas 90] M.P. GASCUEL "Déformations de surfaces complexes : Techniques de haut niveau pour la modélisation et l'animation" - PhD Thesis, Paris-Orsay, octobre 90.

[Luc 90(1)] A. LUCIANI - "Modeles comportementaux : vers une approche instrumentale de la synthèse d'image"- GROPLAN 89 - revue BIGRE + GLOBULE - ler trimestre 90.

[Luc 90(2)] A. LUCIANI - "Physical models in animation : Towards a modular and intrumental approach" - In EUROGRAPHICS Workshop on Animation and Simulation - September 90.

A.C.R.O.E., Ministère de la Culture, Laboratoire d'Informatique Fondamentale et d'Intelligence Artificielle-IMAG-CNRS, 38000 Grenoble, France 